\title{
A planar interdigitated ring electrode array via dielectrophoresis for uniform patterning of cells
}

\author{
Lo-Chang Hsiung a ${ }^{\mathrm{a}}$ Chun-Hui Yang ${ }^{\mathrm{a}}$, Chi-Li Chiu ${ }^{\mathrm{b}}$, Chen-Lin Chen ${ }^{\mathrm{a}}$, Yueh Wang ${ }^{\mathrm{b}}$, \\ Hsinyu Lee ${ }^{\mathrm{b}}$, Ji-Yen Cheng ${ }^{\mathrm{c}}$, Ming-Chih Ho ${ }^{\mathrm{d}}$, Andrew M. Wo ${ }^{\mathrm{a}, *}$ \\ a Institute of Applied Mechanics, National Taiwan University, Taipei 106, Taiwan, ROC \\ b Department of Life Science, National Taiwan University, Taipei 106, Taiwan, ROC \\ ${ }^{c}$ Research Center for Applied Sciences, Academia Sinica, Taipei 115, Taiwan, ROC \\ d Department of Surgery, National Taiwan University Hospital, Taipei 100, Taiwan, ROC
}

\section{A R T I C L E I N F O}

\section{Article history:}

Received 7 April 2008

Received in revised form 27 June 2008

Accepted 9 July 2008

Available online 25 July 2008

\section{Keywords:}

Dielectrophoresis

Electrode array

Cell patterning

Microfluidics

Cellular microarray

\begin{abstract}
A B S T R A C T
Uniform patterning of cells is highly desirable for most cellular studies involving cell-cell interactions but is often difficult in an in vitro environment. This paper presents the development of a collagen-coated planar interdigitated ring electrode (PIRE) array utilizing positive dielectrophoresis to pattern cells uniformly. Key features of the PIRE design include: (1) maximizing length along the edges where the localized maximum in the electric field exists; (2) making the inner gap slightly smaller than the outer gap in causing the electric field strength near the center of a PIRE being generally stronger than that near the outer edge of the same PIRE. Results of human hepatocellular carcinoma cells, HepG2, adhered on a $6 \times 6$ PIRE array show that cells patterned within minutes with good uniformity ( $48 \pm 6$ cells per PIRE). Cell viability test revealed healthy patterned cells after $24 \mathrm{~h}$ that were still confined to the collagen-coated PIREs. Furthermore, quantification of fluorescence intensity of living cells shows an acceptable reproducibility of cell viability among PIREs (mean normalized intensity per PIRE was $1 \pm 0.138$ ). The results suggest that the PIRE array would benefit applications that desire uniform cellular patterning, and improve both response and reproducibility of cell-based biosensors.
\end{abstract}

(c) 2008 Elsevier B.V. All rights reserved.

\section{Introduction}

Microfluidics has become an enabling technology to allow miniaturization of biochemical studies. Small sample volumes, rapid responses, and low cost chip-based platforms with functional integration for analysis have been demonstrated (Dittrich and Manz, 2006; Haeberle and Zengerle, 2007). An example of this is a dielectrophoresis (DEP)-based microfluidic chip (Gascoyne and Vykoukal, 2004; Voldman, 2006), which has been widely used to manipulate bioparticles due to the non-contact nature of the technology. Leveraging the polarizable behavior of most bioparticles, a non-uniform electric field produced locally allows deterministic motion of the bioparticles considered (Yantzi et al., 2007; Zheng et al., 2004). The chip design uses either a single pair of electrodes or, in many cases, an array configuration that organizes bioparticles into groups and often allows high-throughput cellular analysis.

\footnotetext{
* Corresponding author at: Institute of Applied Mechanics, National Taiwan University, No. 1, Sec. 4, Roosevelt Road, Taipei 10617, Taiwan (ROC). Tel.: +88623366 5656; fax: +886223639290.

E-mail address: Andrew@iam.ntu.edu.tw (A.M. Wo).
}

Dielectrophoretic array chips can be roughly categorized into three types according to the way an electric field interacts with the cells, namely, positive dielectrophoretic ( $p$-DEP), negative dielectrophoretic (n-DEP), and a combination of the two. Positive-DEP cell patterning is generally used in chips to attract cells to electrode surfaces for cell patterning. Among p-DEP applications, electrode geometries that were organized in array formats were designed to manipulate single cells (Gray et al., 2004a,b; Taff and Voldman, 2005). In addition to this, p-DEP cell patterning and subsequent "photopatterning" - a technique used to selectively crosslink hydrogel microstructures by UV light exposure - were exploited to construct three-dimensional cellular structures to study the effect of cluster size on cellular biosynthesis (Albrecht et al., 2005, 2006, 2007). Furthermore, a biomimetic patterning of dual types of cells was demonstrated via p-DEP forces (Ho et al., 2006).

In contrast to p-DEP cell patterning, which requires a lowconductive buffer to ensure cells are more polarizable than the surrounding medium, n-DEP can pattern cells in a cell culture medium throughout the patterning process. One application was dielectrophoretic field-flow-fractionation (DEP-FFF), a n-DEP technique that exploits differences in dielectric properties and density of cells (Yang et al., 2000). By using DEP-FFF, mixtures of major 
human leukocyte subpopulations were separated. Another application used n-DEP "virtual" microwells to trap single cells (Mittal et al., 2007). Furthermore, loading and unloading of single cells in a prescribed site was also demonstrated (Taff et al., 2007).

Combination of p-DEP and n-DEP in a DEP array chip is another alternative in manipulating and separating cells. Different types of cells were separated and arrayed by exploiting both p-DEP and nDEP forces (Huang et al., 2002). Leukocyte subpopulations were fractionated from a heterogeneous mixture (Holmes and Morgan, 2004). Moreover, through additional establishment of a stream of both viable and non-viable cells supported by sheath flow, cell fractionation was achieved in a continuous fashion (Li and Kaler, 2004).

While various aforementioned DEP array chips have been applied to pattern cells, several non-DEP approaches for cell patterning have also been made: photolithographic approaches (Bhatia et al., 1999; Britland et al., 1992; Kane et al., 1999; Ostuni et al., 2001), and microarrayer approaches (Anderson et al., 2004; Flaim et al., 2005). However, a common problem of the exsiting techniques in patterning cells is low uniformity of the number of patterned cells, which may have consequences in undesirable cellular responses (Albrecht et al., 2006; Bhatia et al., 1999; Gagne et al., 2000; Iwasa et al., 2003). Therefore, uniform cell patterning is often desired in in vitro cellular studies which involve cell-cell interactions.

This paper reports the development of a planar interdigitated ring electrode (PIRE) array towards uniform patterning of cells in the microfluidic chip. The electric fields generated by PIRE array play a key role in DCP, hence the interplay between DEP force and hydrodynamic force on cell motion is discussed. Then, the performance of PIRE array for providing uniform DCP and cell viability are evaluated. This technique may provide an alternative for manufacturing cellular microarray, and may improve both response and reproducibility of cell-based biosensors.

\section{Design of the PIRE array and simulations}

In this work, we designed a unique electrode array, shown in Fig. 1, to allow uniform and rapid cell patterning via p-DEP. The requirement of uniformity calls for special consideration of the imposed electric fields. First, an interdigitated, ring-shaped electrode which allows maximum length along the edges within an electrode was adopted (see the enlarged view of Fig. 1) since cells are usually patterned along electrode edges where the localized maximum in the electric field exists. Second, rapid patterning was achieved via electrodes with sufficient width that would accelerate cell patterning (Albrecht et al., 2004). Third, the gap region of the interdigitated electrode is crucial in ensuring a quality cellular pattern. In the design, the inner gap of a PIRE is smaller than the outer gap, causing cells to be patterned near the PIRE center first then proceed toward the outer edge of the same PIRE. Fourth, leverage of several features of conventional cellular microarray proves helpful for the microfluidic chip: the diameter of a PIRE and spacing between two neighboring PIREs are chosen to be compatible with widely available microarray analysis platforms. Fifth, the PIRE design requires less inter-connected wiring among electrodes. With the above considerations, the dimensions of the PIRE array were chosen to be $15 \mu \mathrm{m}$ electrode width, $10 \mu \mathrm{m}$ inner gap, $15 \mu \mathrm{m}$ outer gap, $125 \mu \mathrm{m}$ diameter for a single PIRE, and $200 \mu \mathrm{m}$ center-to-center spacing between two neighboring PIREs.

Calculation of the 3D steady-state electric field, with commercial software (COMSOL Multiphysics 3.2), allows optimization of the PIRE design. The geometry used was representative of

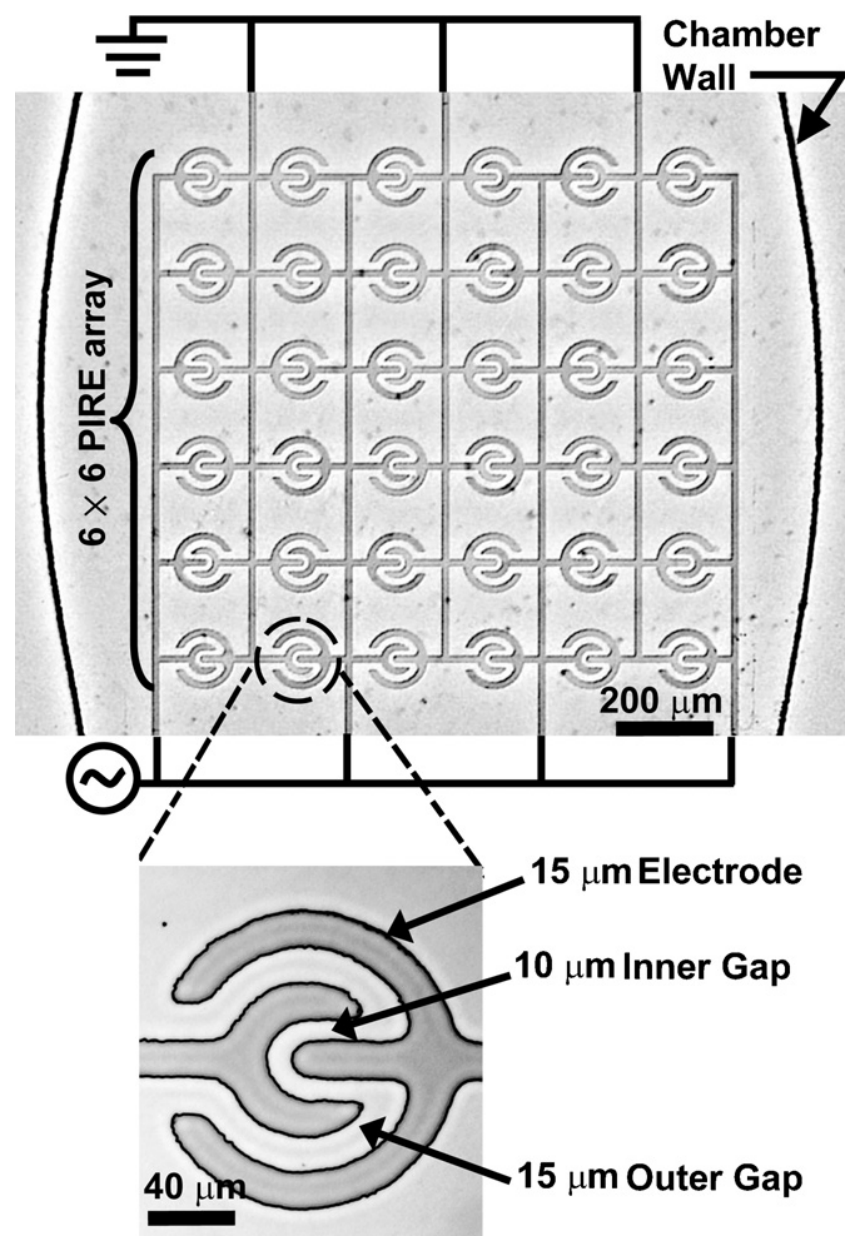

Fig. 1. The $6 \times 6$ planar interdigitated ring electrode (PIRE) array. The PIRE array is designed to pattern cells uniformly via dielectrophoretic cell patterning (DCP).

experimental conditions, with a $50 \mu \mathrm{m}$ chamber height and $200 \mathrm{~nm}$ indium tin oxide (ITO) PIRE thickness. The boundary conditions imposed were: field continuity between different materials, electric insulation on the top and side walls, and applied AC signal of $5 \mathrm{Vpp}$ (peak-to-peak voltage) at $5 \mathrm{MHz}$ on all PIREs. Further, we set the glass substrate's conductivity to $1 \times 10^{-18} \mathrm{~S} / \mathrm{m}$ (siemens per meter) and its relative permittivity to 4.2 . The settings of the DEP buffer were conductivity of $2.31 \times 10^{-2} \mathrm{~S} / \mathrm{m}$, relative permittivity of 80 , and density of $1000 \mathrm{~kg} / \mathrm{m}^{3}$.

\section{Experimental}

\subsection{Chip fabrication and collagen coating}

Microfabrication of the collagen-coated PIRE array chip utilized a combination of photolithography and replica molding. The microfabrication required the following steps (Fig. 2). Firstly, photoresist (S1813, Shipley) was micropatterned on a $210-\mathrm{nm}$ thick ITO film (416-744-X0, Merck) (Fig. 2a). Then, reactive ion etching (RIE) (Plasmalab 80 plus, Oxford) via argon plasma (chamber pressure of $10 \mathrm{mTorr}$, flow rate of $40 \mathrm{sccm}$ (standard cubic centimeters per minute), forward power of $200 \mathrm{~W}$, treatment time of $25 \mathrm{~min}$ ) was used to micromachine the ITO film as PIRE arrays (Fig. 2b). Photoresist was then stripped off using acetone (Fig. 2c). Photoresist was used again in order to micropattern collagen on PIREs (Fig. 2d). 
(a)

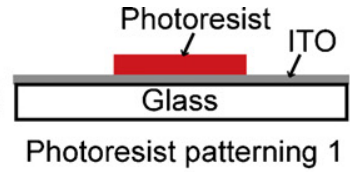

(e)

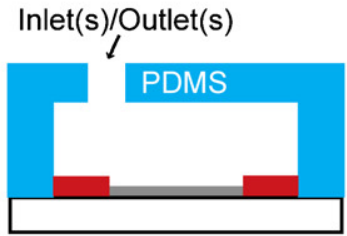

PDMS bonding (b)
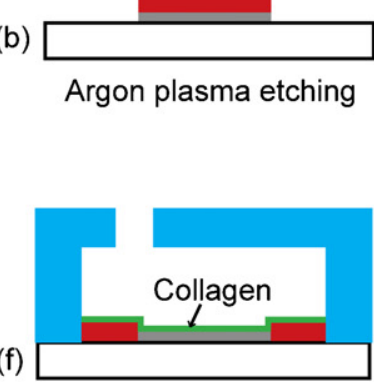

Collagen coating (c)

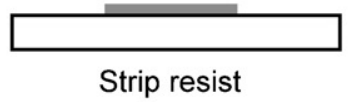

(d)

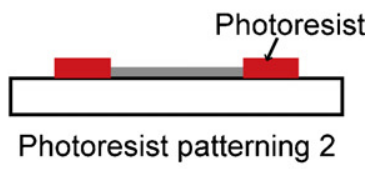

(g)

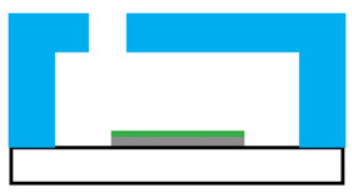

Strip resist

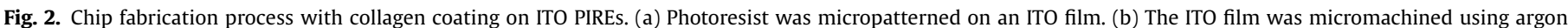

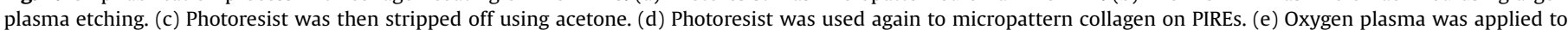

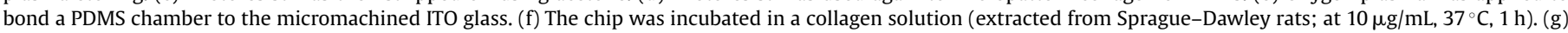

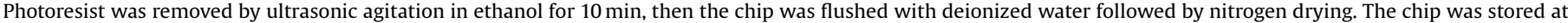
$4{ }^{\circ} \mathrm{C}$ prior to use.

A microchamber with $50 \mu \mathrm{m}$ chamber height was fabricated via soft lithography using poly(dimethylsiloxane) (PDMS) (Sylgard 184, Dow Corning). Then, the molded PDMS was bonded to the micromachined ITO glass following treated with oxygen plasma (Fig. 2e).

To promote cell adhesion and normal cell functions, the PIRE array was coated with collagen. Deionized water was injected into the interior of the microchamber to clean unwanted residuals. Then, the chip was incubated $\left(37^{\circ} \mathrm{C}, 1 \mathrm{~h}\right)$ in $10 \mu \mathrm{g} / \mathrm{mL}$ collagen solution, which was extracted from Sprague-Dawley rats (Folkman et al., 1989; Madri and Williams, 1983) and was mainly constituted of type I collagen (Fig. 2f), to coat PIREs with collagen. Afterwards, photoresist was removed by ultrasonic agitation for $10 \mathrm{~min}$ in ethanol, which may reduce denaturation of the micropatterned collagen comparing to acetone (Fig. $2 \mathrm{~g}$ ). Then, the chip was flushed with deionized water followed by nitrogen drying. Finally, the chip was stored at $4{ }^{\circ} \mathrm{C}$ prior to use.

\subsection{The preparation of cells}

Human hepatocellular carcinoma cells, HepG2 (ATCC, HB-8065), were incubated at $37{ }^{\circ} \mathrm{C}$ under a humidified $5 \% \mathrm{CO}_{2}$ atmosphere. The culture medium was minimum essential medium (MEM) (12561, GIBCO) with 10\% fetal bovine serum (FBS) (SV30014, Hyclone) and 1\% penicillin/streptomycin (15140, GIBCO). Successively, cells were detached using $0.25 \%$ trypsin with EDTA-4Na (tetra-sodium ethylenediaminetetraacetate) (25200, GIBCO). Then, cells were resuspended in the EGTA (ethylene glycol tetraacetic acid)-containing DEP buffer (10 mM HEPES, $55 \mathrm{mM}$ D-glucose, $221 \mathrm{mM}$ sucrose, $0.5 \mathrm{mM}$ EGTA, 1\% penicillin/streptomycin; $\mathrm{pH}$ 7.0, 3000 sm, $2.31 \times 10^{-2} \mathrm{~S} / \mathrm{m}$ ) of a $\sim 5 \times 10^{6}$ cells $/ \mathrm{mL}$ for DCP.

\subsection{Dielectrophoretic cell patterning (DCP)}

The procedure for DCP (Albrecht et al., 2006; Ho et al., 2006) was designed to obtain a uniform and viable cellular pattern. Firstly, suspended cells in the EGTA-containing DEP buffer were seeded into the microchamber with a flow rate of $5 \mu \mathrm{L} / \mathrm{min}$. Then, AC signal ( $5 \mathrm{Vpp}, 5 \mathrm{MHz}$ ) was applied to the PIRE arrays to generate p-DEP forces to pattern cells on PIRE arrays. During the cell seeding process, cells occasionally only flowed through some areas of the $6 \times 6$ PIRE array, which results in large-scale non-uniform patterning (see supplementary data). Therefore, a small vibrator (304-002, Precision Microdrives) was attached to the tubings for cell seeding to agitate the flow of cells and encourage uniformly distributed cells to flow over the entire $6 \times 6$ array. After the flow of the suspended cells became steady, a calcium-containing DEP buffer without EGTA $\left(0.75 \mathrm{mM} \mathrm{CaCl}_{2} ; \mathrm{pH} 7.0,305 \mathrm{Osm}, 2.74 \times 10^{-2} \mathrm{~S} / \mathrm{m}\right)$ was injected at $5 \mu \mathrm{L} / \mathrm{min}$ to sweep away the non-patterned cells and to promote adhesion of cells onto collagen-coated PIREs. When the sweeping flow became steady, the AC signal was removed. After cells were patterned on collagen-coated PIREs, MEM with 10\% FBS and $1 \%$ penicillin/streptomycin was injected at $5 \mu \mathrm{L} / \mathrm{min}$ to replace the calcium-containing DEP buffer. Then, bright field images of the patterned cells were taken via a charge-coupled device (CCD) camera (DP-70, Olympus) installed on an inverted microscope (IX-71, Olympus). From the images, cells were manually counted to analyze the distribution of patterned cells. The data are the mean of four measurements. Finally, the cell-patterned chip was incubated at $37^{\circ} \mathrm{C}$ for $24 \mathrm{~h}$ for the cell viability test.

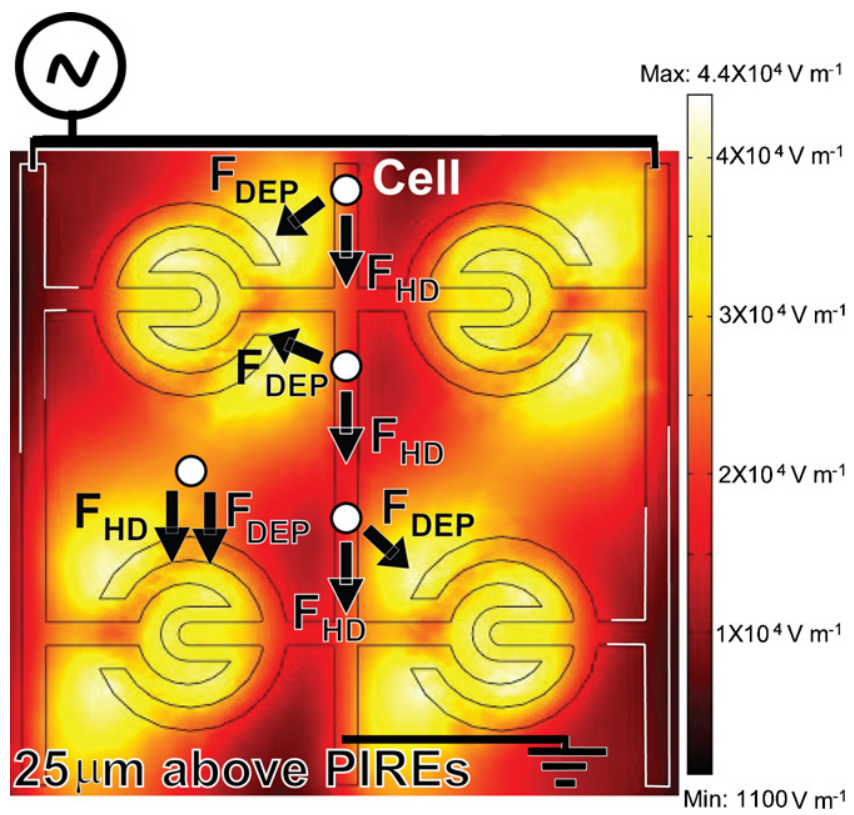

Fig. 3. Simulation of electric field in the region of $2 \times 2$ PIREs ( $5 \mathrm{Vpp}$ at $5 \mathrm{MHz}$ ) The non-uniform electric field of the PIREs is spatially localized. The electric field strength near the center of a PIRE is generally stronger than that near the outer edge of the same PIRE. When cells flow above the PIRE array, they simultaneously experienced hydrodynamic force $\left(\mathrm{F}_{\mathrm{HD}}\right)$ from background flow and positive DEP forces $\left(F_{D E P}\right)$ from the non-uniform electric field. The force balance determines the final cell trajectory. 
(a)

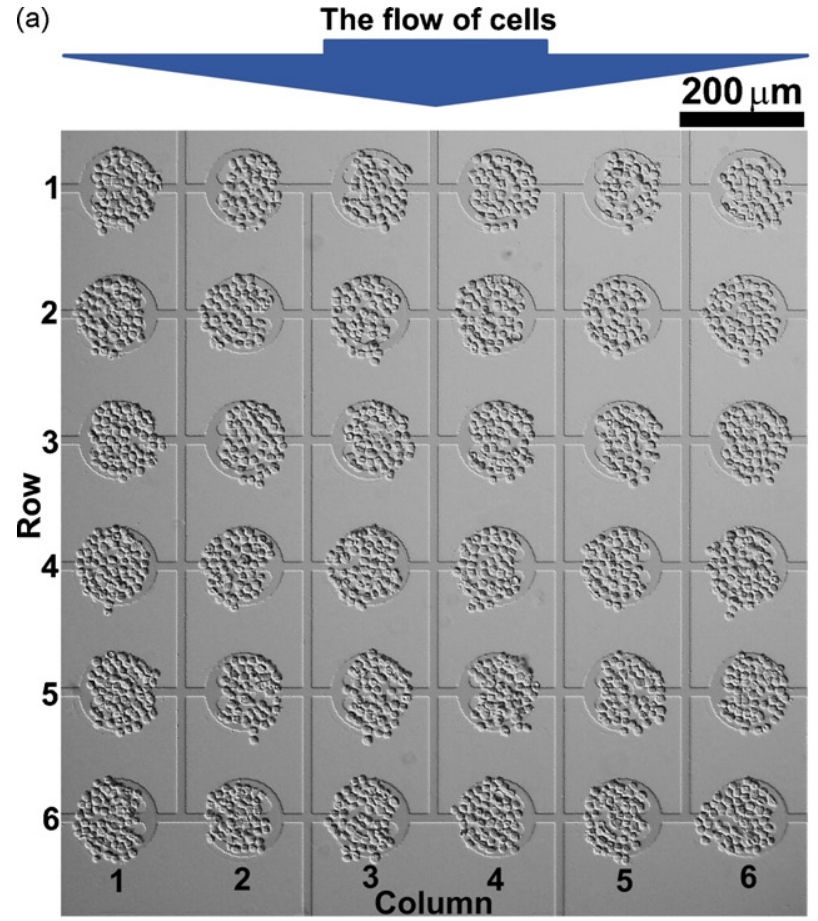

(c)

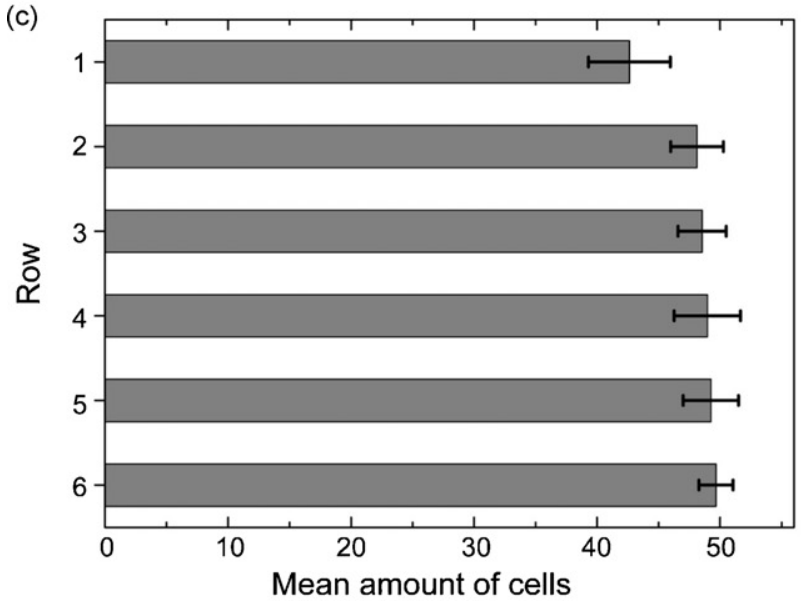

(b)
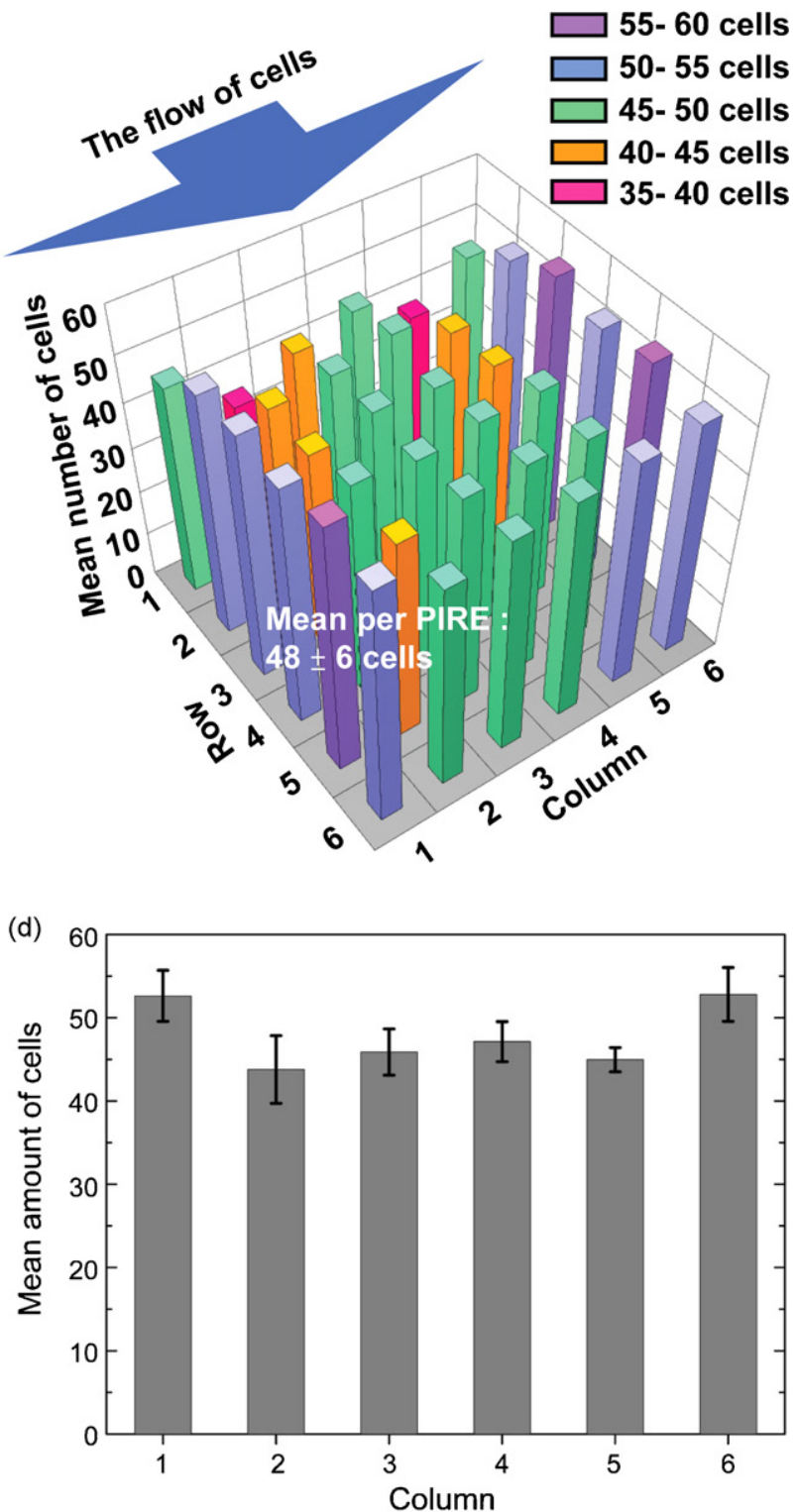

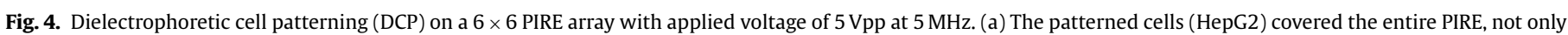

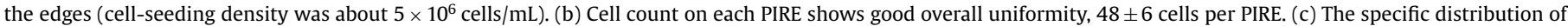
patterned cells in each row of electrode within the PIRE array. (d) The specific distribution of patterned cells in each column of electrode.

\subsection{Cell viability test}

The cell viability of the patterned cells stained with calcein AM (calcein acetoxymethylester) (C3100MP, Invitrogen) was examined after incubation for $24 \mathrm{~h}$. When calcein AM permeates a living cell's plasma membrane, it will be hydrolyzed by the esterase present in the cytoplasm of a living cell to form calcein and emits bright green fluorescence $(515 \mathrm{~nm})$. Firstly, calcein AM was prepared as a stock solution in DMSO $(5 \mathrm{mM})$. Then, the stock solution was diluted with PBS (solution:PBS=1:250). Next, the calcein AM dilution was injected into the microchamber at a flow rate of $5 \mu \mathrm{L} / \mathrm{min}$ to replace the cell culture medium. Then, green fluorescent images and bright field images of the cells were taken via a CCD camera (DP-70, Olympus) on a microscope (IX-71, Olympus).

\subsection{Image processing and analysis}

Viability of patterned cells after $24 \mathrm{~h}$ incubation was estimated using a bright field image of patterned cells overlaid by the associated fluorescent image (processed via the software ImageJ $1.40 \mathrm{~g}$ ). Then, fluorescence images were analyzed (GenePix Pro 6.1) to quantify fluorescence intensities, with the background intensity subtracted from the intensity of living cells. Finally, the mean intensity - the average intensity per pixel within a PIRE - and the associated standard deviation were normalized by the mean intensity, allowing a comparison of the normalized standard deviation among PIREs. The calculated values would be helpful to identify reproducibility of cell viability among PIREs. 
(a)

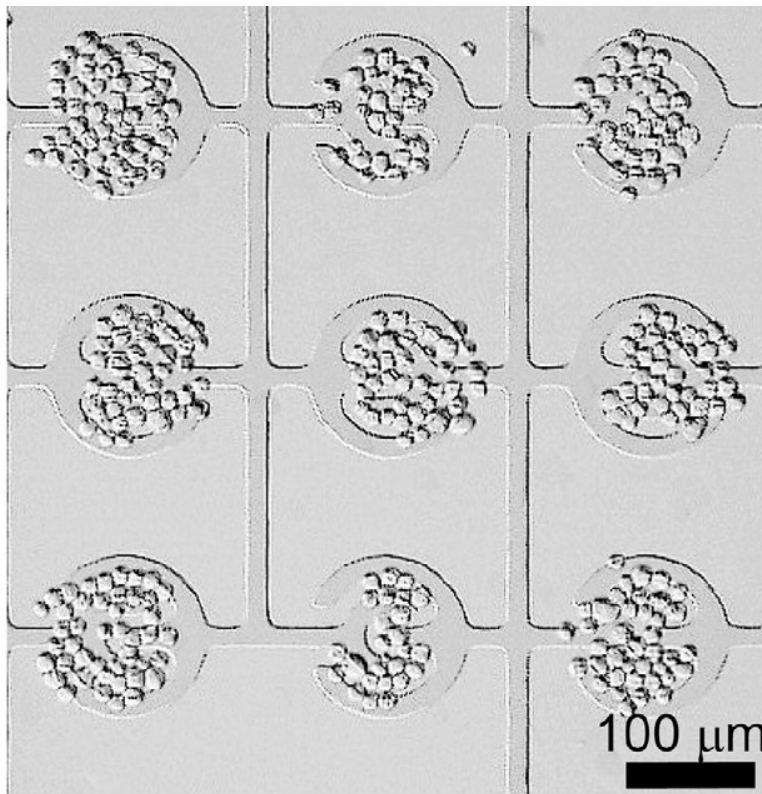

(c)

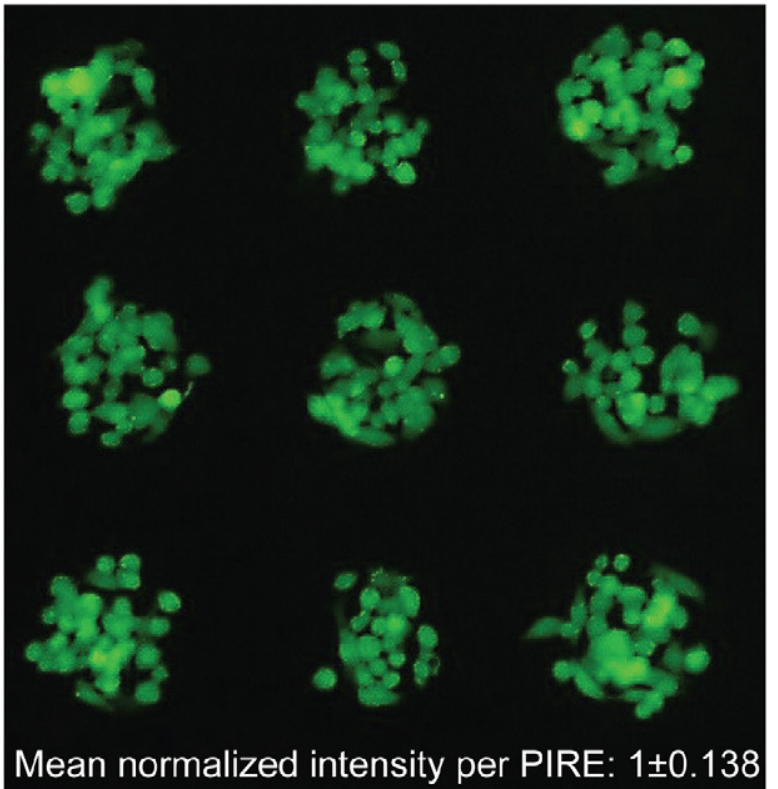

(b)

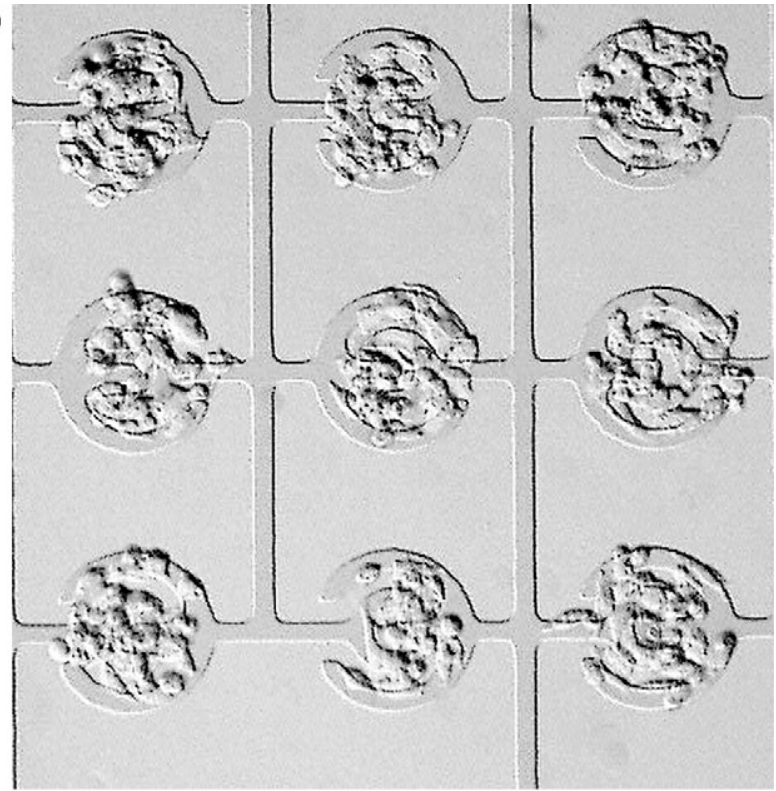

(d)

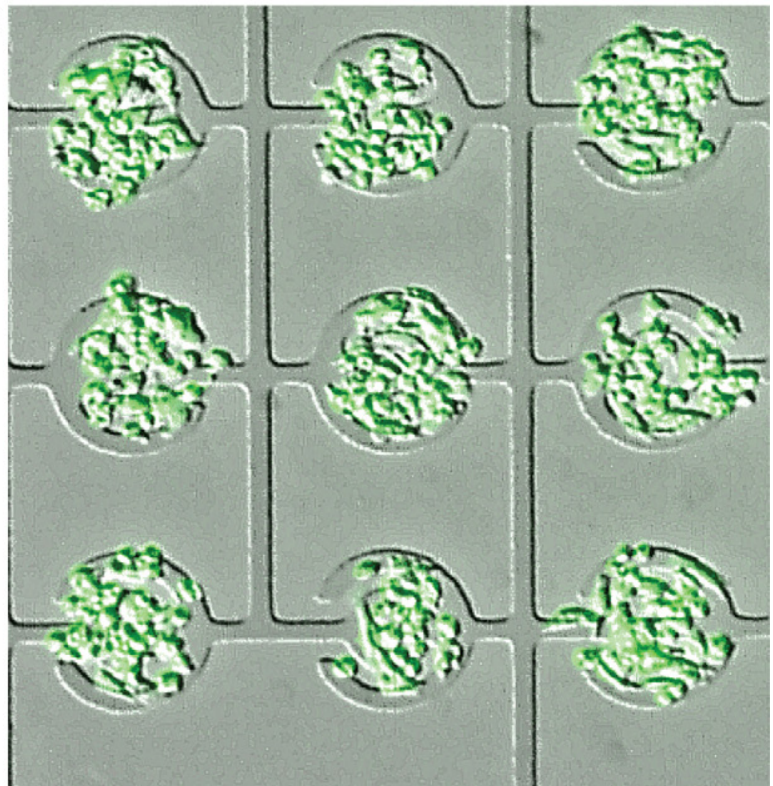

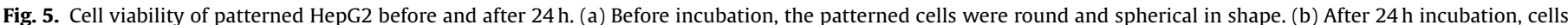

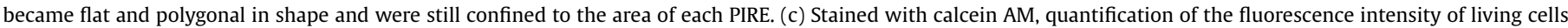

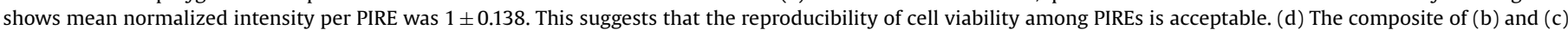
reveals high cell viability by most cells being stained positively.

\section{Results and discussion}

\subsection{Localized electric fields on cell patterning}

Results of simulation of localized electric fields ( $5 \mathrm{Vpp}$ at $5 \mathrm{MHz}$ ) (Fig. 3) represented a stronger non-uniform electric field by yellow (or white in black-and-white printing) and a weaker field by black. The non-uniform electric fields of the PIREs were spatially localized, and the electric field strength near the center of a PIRE was generally stronger than that near the outer edge of the same PIRE, due to the smaller gap near the center. When cells flowed through PIREs, they simultaneously experienced a hydrodynamic force $\left(\mathrm{F}_{\mathrm{HD}}\right)$ from a background flow and positive DEP forces $\left(F_{D E P}\right)$ from the non-uniform electric fields. Prior to cells arriving at PIREs, forces acting on the cells contribute to the directions of resultant forces, pushing them toward PIREs. If a cell is not patterned by a given PIRE, the cell shall be patterned by a downstream PIRE.

\subsection{The performance of $D C P$}

Fig. 4 presents results of patterned HepG 2 on the $6 \times 6$ PIRE array. Fig. 4a shows that the patterned cells covered the entire surface of each PIRE, not only the edges. Cells were uniformly patterned as a cellular microarray within minutes, while hours would be required for other microarrayer approaches. Further, a lack of cell mass was evident in the chip, hence avoiding subsequent damage to patterned cells. This was achieved by removal of free extracellular calcium by adding EGTA (Lin et al., 2006), a chelating agent with a high affinity for calcium ions, since most cell-cell 
adhesion molecules are calcium-dependent (e.g. cadherins, selectins, and integrins).

Overall uniformity of $48 \pm 6$ cells per PIRE for the entire $6 \times 6$ PIRE array was demonstrated in Fig. $4 \mathrm{~b}$. Furthermore, specific distribution of patterned cells among rows (Fig. 4c) reveal cells on the first upstream row had fewer cells than the other rows. This is likely due to the average height of suspended cells entering the array area being higher than that of those further downstream, since the collective effort of p-DEP forces will cause the average height of cells above PIREs to be lowered. About specific cell distribution among columns (Fig. 4d), the number of cells on the first column and sixth column, the columns near chamber walls (see Fig. 1), was larger than one of central columns. The reason is most likely due to the fact that the flow rate near a chamber wall is much slower than that in the central part of the same chamber, as a result of the laminar flow profile in the microchamber (the Reynolds number, based on the average flow speed and the chamber width, was about 2, which was much less then 2000-a generally accepted upper bound for laminar flow (Potter and Wiggert, 2001)). Therefore, one expects the suspended cells above the first column and the sixth column to flow slower, thus causing the cells to be patterned on the first column and the sixth column more easily.

Comparison with other design of DCP (Albrecht et al., 2005, 2006) might be helpful to benchmark the PIRE design. While their emphases were on constructing three-dimensional cellular structures for tissue engineering, the uniformity of patterned cell was specifically documented. The comparison shows the coefficient of variation of the uniformity of cell count in our design appears to be an improvement: coefficient of variation of our design was $12.5 \%$ versus $18.7-52.0 \%$. This improvement was mainly associated with the key features of the PIRE design, namely (1) allowing maximum edge length per PIRE, since the localized maximum in the electric field exists along the edges; (2) encouraging cells to be firstly patterned near the center of a PIRE by making the inner gap (the distance between electrode edges near the center) slightly smaller than the outer gap, causing the electric field strength near the center being slightly stronger than the outer.

The uniformity of DCP may be further improved by several methods. First, one can add electrodes upstream of the PIRE array to generate p-DEP forces for lowering the heights of suspended cells. Thus, the number of cells found on the first row may be close to the number found on any other row. Second, microstructures added in the upstream area might flatten out the flow profile (Yuen et al., 2001) to equalize the hydrodynamic forces on different suspended cells. Third, combining a chaotic mixer might circulate cells to increase the number of cells brought in proximity to PIRE surfaces (Gadish and Voldman, 2006). Last, lowering the flow rate would allow cells to suspend above PIREs for a longer time to be attracted unto PIRE surfaces.

\subsection{Cell viability}

High cell viability ensures efficacy of the design. Results of viability of HepG2 at $24 \mathrm{~h}$ are shown in Fig. 5. Before incubation, the patterned cells were round and spherical in shape (Fig. 5a). However, after $24 \mathrm{~h}$ incubation, the cells became flat and polygonal in shape and were still confined to collagen-coated PIREs (Fig. 5b). The confined cells on the collagen-coated PIRE array (Fig. 5b) would facilitate cellular analysis (e.g. microarray analysis). Besides, high cell viability was demonstrated by most cells being stained positively with calcein AM after incubation (Fig. 5c and d). Quantification of fluorescence intensity of living cells in Fig. 5c shows mean normalized intensity per PIRE was $1 \pm 0.138$ (coefficient of variation was $13.8 \%$ ). This suggests that the reproducibility of cell viability among PIREs is acceptable. The high cell viability may be attributed to the following factors: (1) rapid cell patterning reducing the electric field-induced damage of cells (Glasser and Fuhr, 1998); (2) low conductivity of the DEP buffer $\left(2.31 \times 10^{-2} \mathrm{~S} / \mathrm{m}\right)$ reducing current-induced joule heating (Glasser and Fuhr, 1998); (3) penicillin/streptomycin in the DEP buffer reducing contamination during DCP; (4) the meticulously prepared DEP buffer (osmolarity 300 Osm, pH value 7.0) finely adjusted to physiological conditions of mammalian cells. On the contrary, if the aforementioned procedure for DCP is not strictly followed, the consequent cell viability will be conspicuously declined (see supplementary data).

\section{Conclusions}

This study puts forth the development of a planar interdigitated ring electrode (PIRE) array that results in uniform cell patterning ( $48 \pm 6$ cells per PIRE) with proven cell viability. The tailored, non-uniform electric field attracting suspended cells to the collagen-coated electrodes is the key to the success. This tuned, positive dielectrophoretic-based approach may improve both response and reproducibility of cell-based biosensors, and should minimize cell waste for applications that involve precious samples from patients. The next step of this study is to utilize the PIRE array chip to manufacture cellular microarray for assessing the efficacy of a particular chemotherapeutic regimen on a patient's sample prior to treatment.

\section{Acknowledgement}

Funding from the National Science Council (grant NSC 94-2212E-002-054) is gratefully acknowledged.

\section{Appendix A. Supplementary data}

Supplementary data associated with this article can be found, in the online version, at doi:10.1016/j.bios.2008.07.027.

\section{References}

Albrecht, D.R., Sah, R.L., Bhatia, S.N., 2004. Biophys. J. 87 (4), 2131-2147.

Albrecht, D.R., Tsang, V.L., Sah, R.L., Bhatia, S.N., 2005. Lab Chip 5 (1), 111-118.

Albrecht, D.R., Underhill, G.H., Wassermann, T.B., Sah, R.L., Bhatia, S.N., 2006. Nat Methods 3 (5), 369-375.

Albrecht, D.R., Underhill, G.H., Mendelson, A., Bhatia, S.N., 2007. Lab Chip 7 (6), 702-709.

Anderson, D.G., Levenberg, S., Langer, R., 2004. Nat. Biotechnol. 22 (7), 863-866.

Bhatia, S.N., Balis, U.J., Yarmush, M.L., Toner, M., 1999. FASEB J. 13 (14), $1883-$ 1900.

Britland, S., Perezarnaud, E., Clark, P., McGinn, B., Connolly, P., Moores, G., 1992 Biotechnol. Prog. 8 (2), 155-160.

Dittrich, P.S., Manz, A., 2006. Nat. Rev. Drug Discov. 5 (3), 210-218.

Flaim, C.J., Chien, S., Bhatia, S.N., 2005. Nat. Methods 2 (2), 119-125.

Folkman, J., Watson, K., Ingber, D., Hanahan, D., 1989. Nature 339 (6219), 58-61.

Gadish, N., Voldman, J., 2006. Anal. Chem. 78 (22), 7870-7876.

Gagne, T.A., Chappell-Afonso, K., Johnson, J.L., McPherson, J.M., Oldham, C.A., Tubo, R.A., Vaccaro, C., Vasios, G.W., 2000. J. Orthop. Res. 18 (6), 882-890.

Gascoyne, P.R.C., Vykoukal, J.V., 2004. Proc. IEEE 92 (1), 22-42.

Glasser, H., Fuhr, G., 1998. Bioelectrochem. Bioenerg. 47 (2), 301-310.

Gray, D.S., Tan, J.L., Voldman, J., Chen, C.S., 2004a. Biosens. Bioelectron. 19 (7), 771-780.

Gray, D.S., Tan, J.L., Voldman, J., Chen, C.S., 2004b. Biosens. Bioelectron. 19 (12), 1765-1774.

Haeberle, S., Zengerle, R., 2007. Lab Chip 7 (9), 1094-1110.

Ho, C.T., Lin, R.Z., Chang, W.Y., Chang, H.Y., Liu, C.H., 2006. Lab Chip 6 (6), 724 734.

Holmes, D., Morgan, H., 2004. Electrostatics 2003. Iop Publishing Ltd., Bristol, pp. 107-112.

Huang, Y., Joo, S., Duhon, M., Heller, M., Wallace, B., Xu, X., 2002. Anal. Chem. 74 (14), 3362-3371 
Iwasa, J., Ochi, M., Uchio, Y., Katsube, K., Adachi, N., Kawasaki, K., 2003. Artif. Organs 27 (3), 249-255.

Kane, R.S., Takayama, S., Ostuni, E., Ingber, D.E., Whitesides, G.M., 1999. Biomaterials 20 (23-24), 2363-2376.

Li, Y.L., Kaler, K., 2004. Anal. Chim. Acta 507 (1), 151-161.

Lin, R.Z., Chou, L.F., Chien, C.C.M., Chang, H.Y., 2006. Cell Tissue Res. 324 (3), 411 422.

Madri, J.A., Williams, S.K., 1983. J. Cell Biol. 97 (1), 153-165.

Mittal, N., Rosenthal, A., Voldman, J., 2007. Lab Chip 7 (9), 1146-1153.

Ostuni, E., Chen, C.S., Ingber, D.E., Whitesides, G.M., 2001. Langmuir 17 (9) 2828-2834.

Potter, M.C., Wiggert, D.C., 2001. Mechanics of Fluids, 3rd ed. Brooks/Cole, California.
Taff, B.M., Desai, S.P., Voldman, J., 2007. The 11th International Conference on Miniaturized Systems for Chemistry and Life Sciences (MicroTAS 2007), Paris, pp. $8-10$.

Taff, B.M., Voldman, J., 2005. Anal. Chem. 77 (24), 7976-7983.

Voldman, J., 2006. Annu. Rev. Biomed. Eng. 8, 425-454.

Yang, J., Huang, Y., Wang, X.B., Becker, F.F., Gascoyne, P.R.C., 2000. Biophys. J. 78 (5), 2680-2689.

Yantzi, J.D., Yeow, J.T.W., Abdallah, S.S., 2007. Biosens. Bioelectron. 22 (11), 2539-2545.

Yuen, P.K., Kricka, L.J., Fortina, P., Panaro, N.J., Sakazume, T., Wilding, P., 2001. Genome Res. 11 (3), 405-412.

Zheng, L.F., Brody, J.P., Burke, P.J., 2004. Biosens. Bioelectron. 20 (3), 606-619. 